\title{
Patient experience of CT colonography and colonoscopy after fecal occult blood test in a national screening programme
}

\author{
Andrew A. Plumb ${ }^{1}$ - Alex Ghanouni ${ }^{2}$ - Colin J. Rees ${ }^{3}$ Paul Hewitson ${ }^{4}$. \\ Claire Nickerson $^{5}$ • Suzanne Wright ${ }^{5}$ - Stuart A. Taylor ${ }^{1}$ • Steve Halligan ${ }^{1}$. \\ Christian von Wagner ${ }^{2}$
}

Received: 9 December 2015 /Revised: 4 March 2016 / Accepted: 20 May 2016/Published online: 10 June 2016

(C) The Author(s) 2016. This article is published with open access at Springerlink.com

\begin{abstract}
Objective To investigate patient experience of CT colonography (CTC) and colonoscopy in a national screening programme.

Methods Retrospective analysis of patient experience postal questionnaires. We included screenees from a fecal occult blood test (FOBt) based screening programme, where CTC was performed when colonoscopy was incomplete or deemed unsuitable. We analyzed questionnaire responses concerning communication of test risks, test-related discomfort and posttest pain, as well as complications. CTC and colonoscopy responses were compared using multilevel logistic regression. Results Of 67,114 subjects identified, 52,805 (79\%) responded. Understanding of test risks was lower for CTC $(1712 / 1970=86.9 \%)$ than colonoscopy $(48783 /$ $50975=95.7 \%, p<0.0001)$. Overall, a slightly greater proportion of screenees found CTC unexpectedly uncomfortable $(506 / 1970=25.7 \%)$ than colonoscopy $(10,705 / 50,975=$ $21.0 \%, p<0.0001)$. CTC was tolerated well as a completion procedure for failed colonoscopy (unexpected discomfort; $\mathrm{CTC}=26.3 \%$ : colonoscopy $=57.0 \%, p<0.001)$. Post-
\end{abstract}

Andrew A. Plumb

andrew.plumb@ucl.ac.uk

1 Centre for Medical Imaging, Division of Medicine, University College London, 3rd Floor East, 250 Euston Road, London NW1 2PG, UK

2 Health Behaviour Research Centre, Department of Epidemiology and Public Health, University College London, London, UK

3 Durham University School of Medicine, Pharmacy and Health, Durham, UK

4 Health Services Research Unit, Nuffield Department of Population Health, University of Oxford, Oxford, UK

5 NHS Cancer Screening Programmes, Fulwood House, Sheffield, UK procedural pain was equally common (CTC: 288/1970, $14.6 \%$, colonoscopy: $7544 / 50,975,14.8 \% ; p=0.55$ ). Adverse event rates were similar in both groups (CTC: 20/ $2947=1.2 \%$; colonoscopy: $683 / 64,312=1.1 \%$ ), but generally less serious with CTC.

Conclusions Even though CTC was reserved for individuals either unsuitable for or unable to complete colonoscopy, we found only small differences in test-related discomfort. CTC was well tolerated as a completion procedure and was extremely safe. CTC can be delivered across a national screening programme with high patient satisfaction.

Key Points

- High patient satisfaction at CTC is deliverable across a national screening programme.

- Patients who cannot tolerate screening colonoscopy are likely to find CTC acceptable.

- CTC is extremely safe; complications are rare and almost never serious.

- Patients may require more detailed information regarding the expected discomfort of CTC.

Keywords Colon cancer - Computed tomography · Endoscopy $\cdot$ Fecal occult blood test $\cdot$ CT colonography (CTC)

\section{Introduction}

Patient experience is fundamental to high-quality healthcare, and is important to patients [1] and policy-makers [2]. Although healthcare services are increasingly scrutinized, many quality assessments overlook patient experience. This is particularly important for screening programmes, as they are predicated on high uptake and acceptability. Mass screening for colorectal cancer (CRC) has been introduced in many countries [3], most often using fecal occult blood testing 
(FOBt) followed by colonoscopy for those testing positive [4, 5]. This reduces CRC-related mortality, proven by randomized trials [6].

In a proportion of individuals, it is not possible to intubate the entire colon-whether due to frailty, technical difficulty, or simply refusal. In this situation, one alternative is to offer CT colonography (CTC), since it is highly sensitive for CRC and polyps both as a primary screening tool $[7,8]$ and in FOBtpositive individuals [9]. Pan-European consensus recommends CTC when colonoscopy is incomplete or not possible [10-12]. Relatively little is known regarding the relative acceptability of CTC versus colonoscopy in this setting.

CTC is generally well-tolerated in comparison to colonoscopy [13-15]. Two randomized studies, one of asymptomatic screenees [16] and one of symptomatic older patients [17] reported similar acceptability of CTC and colonoscopy, with the balance marginally in favour of colonoscopy for screenees [18] and CTC for symptomatic patients [19]. However, it is unknown whether these findings are replicated in routine clinical practice beyond randomized trials. Furthermore, the relevance of these findings to FOBt-based programmes, in which direct colonic investigation is a second-stage test, is also unknown.

Here we describe patient experience of CT colonography in a national FOBt-based CRC screening programme when colonoscopy is not possible or incomplete.

\section{Methods}

\section{Ethical permissions}

Permission to access anonymized, routinely-coded data was granted by the English Bowel Cancer Screening Programme. Approval for evaluation of retrospective, anonymized data was granted by our institutional research office.

\section{Participants}

The English Bowel Cancer Screening Programme (BCSP) is a national screening programme using biennial FOBt and colonoscopy for those testing positive [20]. Eligible residents aged 60-74 years are mailed a FOBt kit, and those testing positive are invited for a clinical assessment and colonoscopy at a local hospital "screening centre". CTC is substituted if colonoscopy is judged inappropriate or infeasible (e.g. due to co-morbidity) or if colonoscopy is incomplete (e.g. impassable tumour/stricture, technical failure). All patients are assessed by a trained healthcare professional prior to colonoscopy or CTC, and the decision to refer to CTC rather than colonoscopy due to unsuitability for the latter is ratified by a screening-accredited colonoscopist. All participants undergoing a colonic test are sent a standard questionnaire 30 days after the process. Here we present data for screenees tested between January 1st, 2011, and December 31st, 2012, (the first two full calendar years after programme roll-out) and focus on CTC-related outcomes; colonoscopy-specific data will be reported elsewhere.

\section{Procedures}

CTC requirements include dual patient positioning, fecal tagging, carbon dioxide insufflation, multislice scanners, trained staff, and a CTC workstation. There are no stipulations regarding use or quantity of purgation; recent survey data suggest the commonest strategy is to use either two sachets of sodium picosulfate/magnesium citrate (Picolax, Ferring Pharmaceuticals, West Drayton, UK) or 100-150 mL of sodium diatrizoate/meglumine diatrizoate (Gastrografin, Bayer plc, Newbury, UK) as a combined purgation/tagging agent. Use of intravenous contrast is discouraged unless specifically needed (e.g. for tumour staging). Radiologists must interpret $>100$ cases per annum (both screening and symptomatic) before commencing work for the programme [12]. Sixty percent of centres perform $>300$ CTC per year [21]. All programme colonoscopies are performed by screening-accredited colonoscopists, a process requiring (a) completion of $>1000$ colonoscopies, (b) success in a written multiple-choice question examination, and (c) successful evaluation of two observed colonoscopies by an external assessor [22]. Sedoanalgesia is administered as required.

\section{Outcome measures}

The patient questionnaire addresses the screening invitation, the pre-colonic test assessment, the preparation for their colonic test, the test itself (including discomfort), post-test symptoms, communication of results, and privacy/dignity. Most items are answered with a five-point Likert-type scale ("strongly agree" to "strongly disagree") or a binary "yes/ no" response.

\section{Data recording and extraction}

We used the BCSP database to extract: participant age, sex, date of screening, screening centre used, screening round (i.e. number of times previously screened via FOBt), colonic test performed, test result, need for any subsequent tests (e.g. colonoscopy following a positive CTC result), recorded complications (entered by screening programme staff), and questionnaire results. Socioeconomic deprivation was estimated using the index of multiple deprivation (IMD) from 2007, a composite deprivation measure linked to postal (ZIP) code. Complications for CTC were divided into direct (i.e. attributed to CTC) and downstream (i.e. after an additional test following (TC). 


\section{Data analysis}

Data were coded using SPSS v21 for Windows (IBM Corp, Armonk, NY, USA) and analyzed with R version 3.0.1 for Mac (R Foundation for Statistical Computing, Austria, Vienna). Participants not responding to any questionnaire item were excluded from the patient experience analysis but were included when calculating complication rates. Missing questionnaire data for those who responded to at least one questionnaire item were handled via 10 multiple imputations using the mice package for $\mathrm{R}$ under the missing at random assumption [23]. Variables used in the imputation model were patient age, sex, socio-economic deprivation, screening round, test used, screening outcome, and questionnaire results.

Following imputation, questionnaire data with a Likerttype scale were collapsed into binary categories of "agreement" and "non-agreement" because initial analysis showed the data violated the proportional odds assumption. Subsequently, multilevel binary logistic regression was used, with the diagnostic test being performed (i.e. CTC or colonoscopy) as the explanatory variable. Covariates in the regression model were the same as those used for the imputation model, although the occurrence of complications and other questionnaire responses were excluded. The model accounted for clustering of screenees into screening centres. All statistical tests were conducted on each multiply imputed data set and pooled according to Rubin [24].

Since we expected that screenees undergoing CTC following colonoscopy (i.e. incomplete colonoscopy) and those triaged directly for CTC (i.e. judged unsuitable for colonoscopy) may have had differing experience, we compared these subgroups. Additionally, we expected that those who underwent colonoscopy following CTC were more likely to require polypectomy (contingent on a CTC abnormality), which increases procedural complexity; hence, subgroup analysis was also performed here.

CTC questionnaire results for individual centres were compared using funnel plots constructed with exact binomial control limits at 2 and 3 standard deviations [25]. We also used logistic regression to examine whether centres using nonlaxative preparation for CTC (data taken from reference [21]) had differences in test discomfort.

\section{Results}

Of 67,114 potentially eligible screenees, 52,805 returned a questionnaire $(78.7 \%$ overall; $51,554 / 65,197=79.1 \%$ for colonoscopy; 2018/2946 $=68.5 \%$ for CTC; Fig. 1). Of these, 603 were excluded due to administrative or data entry errors (incorrect questionnaire mailed, or questionnaire date before test date). One hundred and ninety-seven individuals had no record of undergoing either CTC or colonoscopy. Ultimately,
52,202 questionnaires were analysed, 50,975 for colonoscopy and 1,970 for CTC (Fig. 1).

Demographics and screening characteristics are summarised in Table 1. Slightly more respondents $(1191 / 1970=60.0 \%)$ underwent CTC because of contraindications to colonoscopy rather than incomplete colonoscopy (779/1970, 40.0\%). Individuals who underwent CTC were older (CTC: mean 66.9 years; colonoscopy: mean 66.3 years, $p<0.0001)$ and more likely to be female (CTC: $1017 / 1970=51.6 \%$ female; Colonoscopy: $21,111 / 50975=41.4 \%, p<0.0001$, Table 1). Average socio-economic deprivation was greater for those who underwent CTC (median deprivation $=48$ th percentile) than colonoscopy (median deprivation $=42$ nd percentile), with no difference between the CTC subgroups.

Relatively few responses were missing, ranging from 0.6$15.6 \%$ for the different questionnaire items. There were no differences between complete-case analysis and multiply imputed analysis for any items; multiply imputed responses are reported here, with complete-case data shown in Table 2.

\section{Items regarding pre-test experience}

Satisfaction with the communication of risks and benefits of CTC was high. Respondents agreed or strongly agreed they understood both risks $(1712 / 1970=86.9 \%)$ and benefits $(1844 / 1970=93.6 \%)$ of CTC. Understanding was slightly higher for colonoscopy, with 48,783/50,975 = 95.7 \% agreeing they had understood its risks (odds ratio $[\mathrm{OR}]=3.01,95 \% \mathrm{CI}$ 2.59-3.51, $p<0.0001)$ and 50,057/50,975 = 98.2 \% understanding benefits $(\mathrm{OR}=3.31,95 \% \mathrm{CI} 2.61-4.19, p<0.0001)$. Respondents found bowel preparation instructions clear, for both CTC $(1875 / 1970=95.2 \%$ agreement $)$ and colonoscopy $(49,905 / 50,975=97.9 \%)$, with a small, but statistically significant difference in favour of colonoscopy $(\mathrm{OR}=1.89,95 \% \mathrm{CI}$ 1.50-2.40, $p<0.0001$, Table 2).

\section{Items regarding the test procedure}

Approximately one quarter $(506 / 1970=25.7 \%)$ of respondents found CTC more uncomfortable than expected. This was a larger proportion than for colonoscopy $(10,705 / 50$, $975=21.0 \%$; OR $=0.81,95 \%$ CI 0.72-0.91, $p<0.0001)$. This difference remained even for patients who denied receiving sedation during their colonoscopy (Table 3 ). There was no significant difference in asking for the test to be stopped/paused (CTC: 114/1970 =5.8\%; colonoscopy: $2600 / 50,975=5.1 \%, p=0.28)$, whether or not patients reported receiving sedation for their colonoscopy (Table 3). Almost all individuals agreed they had been treated with both privacy and respect for both tests, although again there were small, but statistically significant differences in favour of colonoscopy (Table 2). 


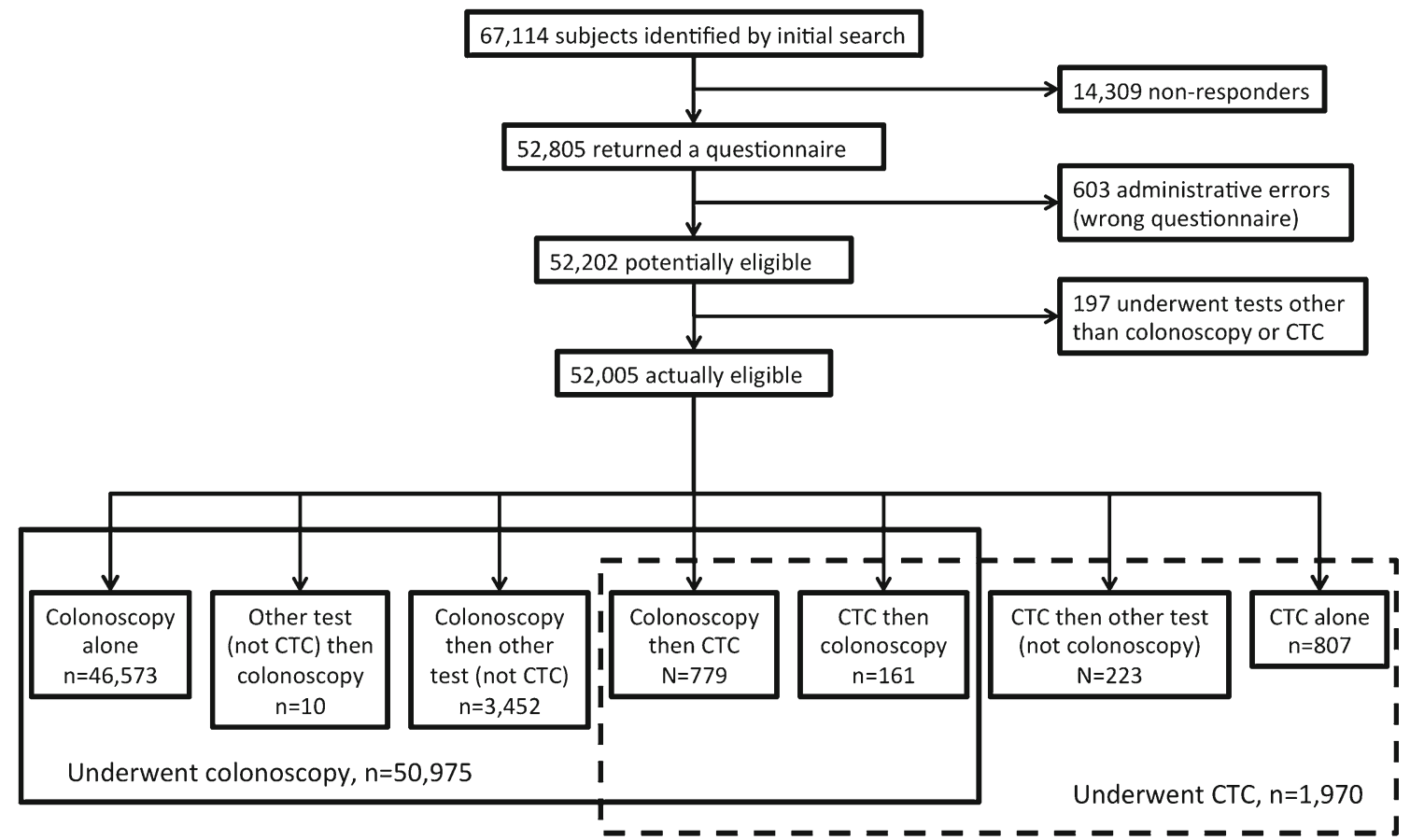

Fig. 1 Study flowchart of inclusions and exclusions (also see text). The term "other test" refers to flexible sigmoidoscopy, barium enema, and non-colonographic abdominopelvic CT scanning, all of which were occasionally used in the programme during the study period (the latter two tests have since been removed as testing options)

\section{Items regarding the post-test experience}

There was no significant difference in the proportion of respondents who recalled suffering rectal/abdominal pain following their diagnostic test (CTC: 288/1970,14.6\%, colonoscopy: $7544 / 50,975,14.8 \% ; p=0.55)$. Again, there was no discernable effect of sedation on this questionnaire item (Table 3). Those who had undergone CTC were less likely to have received their results within seven days $(1564 / 1970=79.4 \%)$ than for colonoscopy $(42,105 / 50$, $975=82.6 \% ; p<0.0001)$. A greater proportion of those who underwent colonoscopy agreed they understood their results $(49,395 / 50,975=96.9 \%)$ than for CTC $(1783 / 1970=90.5 \% ; \mathrm{OR}=3.26,95 \%$ CI 2.71-3.91, $p<0.0001$; Table 2).

\section{Variation by indication for CTC}

There were few differences between questionnaire results for the two CTC subgroups (Table 4). Only the assessment of clarity of bowel preparation and signing of informed consent were significantly different, both judged superior for the group with prior incomplete colonoscopy. Specifically, unexpected discomfort and post-test anorectal/abdominal pain were no different between the two CTC subgroups.

\section{CTC performed when colonoscopy judged unsuitable}

Of the 1191 screenees who underwent CTC after being judged unsuitable for colonoscopy, 161 underwent a subsequent colonoscopy (to investigate CTC-diagnosed abnormality). These individuals reported no significant difference in discomfort between their CTC (30/ $161=18.6 \%$ experienced greater than expected discomfort) and colonoscopy procedures $(32 / 161=19.9 \%$, $p=0.89$ ). Similarly, there was no significant difference in rates of asking for the test to be stopped or paused (CTC: $6 / 161=3.7 \%$, colonoscopy: 5/161 $=3.1 \%$, $p>0.99$ ) or post-test anorectal/abdominal pain (CTC: $21 /$ $161=13.0 \%$; colonoscopy: $24 / 161=14.3 \%, p=0.75$ ).

\section{CTC performed after incomplete colonoscopy}

Screenees who had CTC following colonoscopy (using CTC as a completion test) completed 779 questionnaires. A significantly greater proportion of this subgroup found colonoscopy more uncomfortable than expected when compared with CTC (CTC: 205/779, $26.3 \%$; colonoscopy: $444 / 779,57.0 \%, p<0.001)$. Similarly, these individuals reported more anorectal/abdominal pain after colonoscopy $(187 / 779,24.0 \%)$ than after CTC (108/779, $13.9 \%, p<0.001$, Fig. 2 ). 


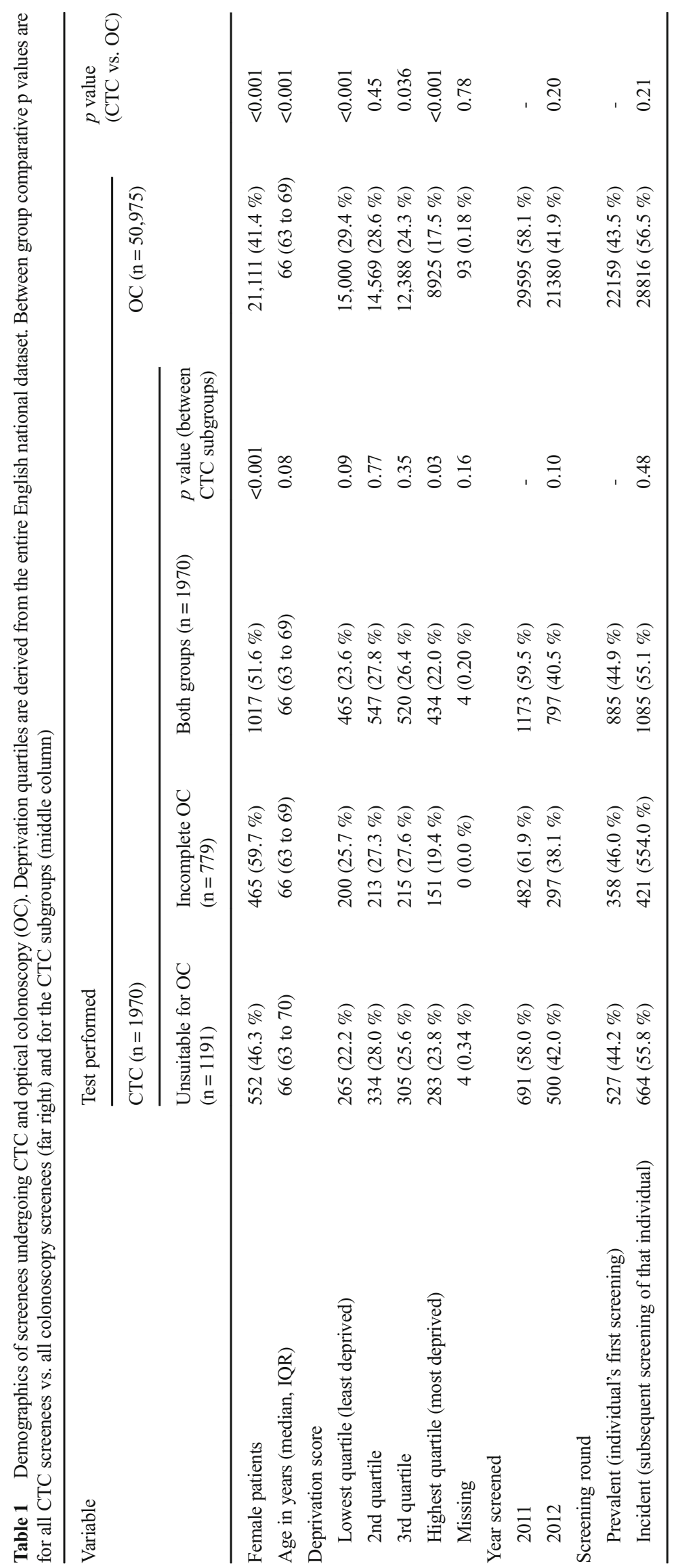




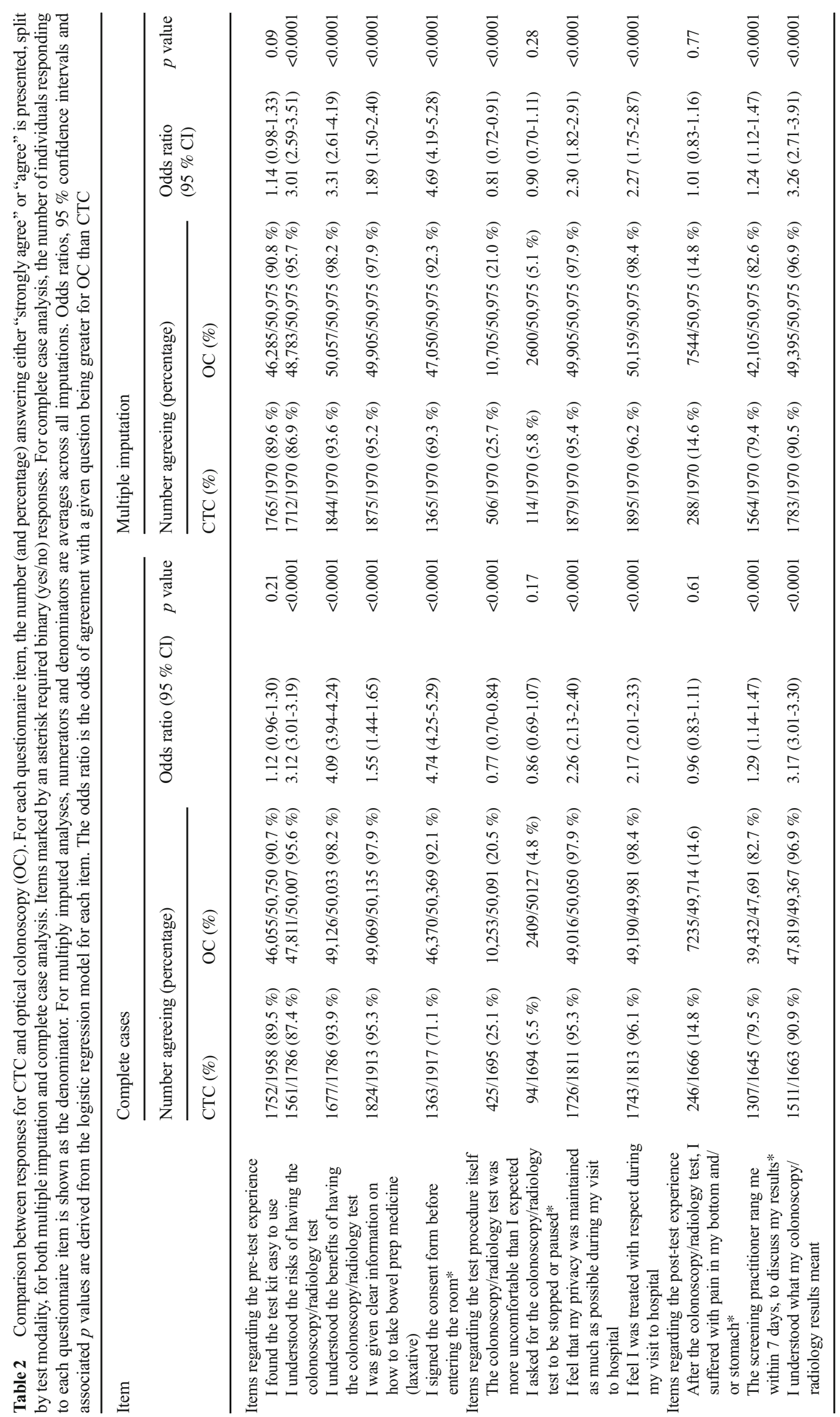




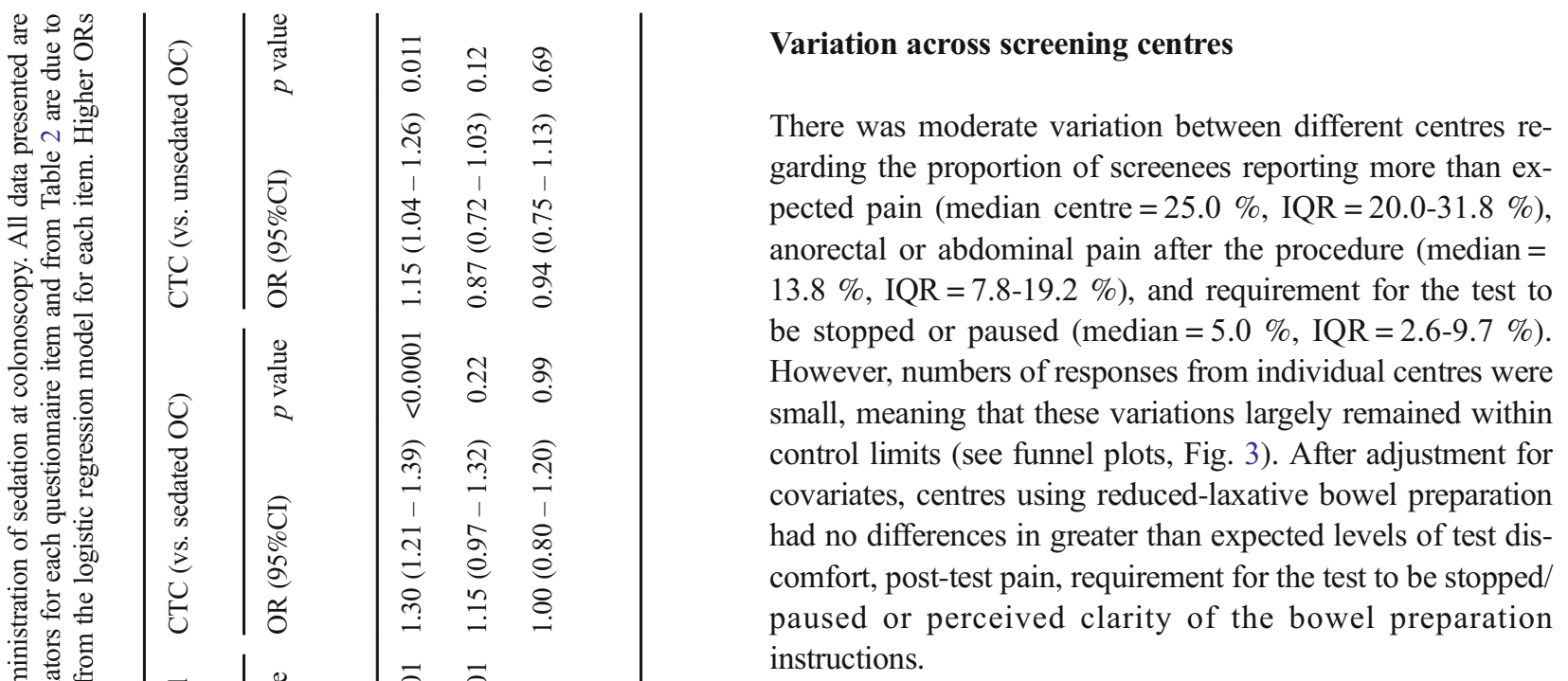

\section{Post-procedural complications}

Data regarding complications were available for 2947 individuals (2988 CTC examinations). Fifteen screenees had complications recorded, which were attributed to CTC (nine severe pain, five adverse reactions to bowel preparation or intravenous medication, and one venous thromboembolism), giving a per-test and per-screenee complication rate of $0.5 \%$. An additional 20 screenees experienced complications ( 15 severe pain, five with bleeding) as a result of further tests provoked by abnormal or incomplete CTC, giving a total complication rate (including downstream complications) of $1.2 \%$. No patient had perforation. A total of 66,783 colonoscopies were performed in 64,312 individuals, of whom 683 had complications, corresponding to a per-test rate of $1.0 \%$ and a perpatient rate of $1.1 \%$. However, more serious complications were recorded after colonoscopy, including 34 perforations, 10 cardiac arrhythmias, and two respiratory arrests.

\section{Discussion}

Colonoscopy and CTC are the preferred methods of pancolonic investigation for screening and symptomatic patients. Most reports of large-scale implementation have focused on detection rates and safety [20,26, 27], but patient experience is also an important facet. Using self-reported patient questionnaires, we documented patient experience in a national FOBt-based CRC screening programme employing CTC in accordance with international consensus $[10,11]$. Overall satisfaction was high for both tests, although CTC was slightly more likely to be judged unexpectedly uncomfortable than was colonoscopy. There was no difference in the rate of intolerable symptoms requiring either test to be stopped or paused, or in anorectal/abdominal pain after the procedure. Complication rates were similar, but less serious after CTC. 


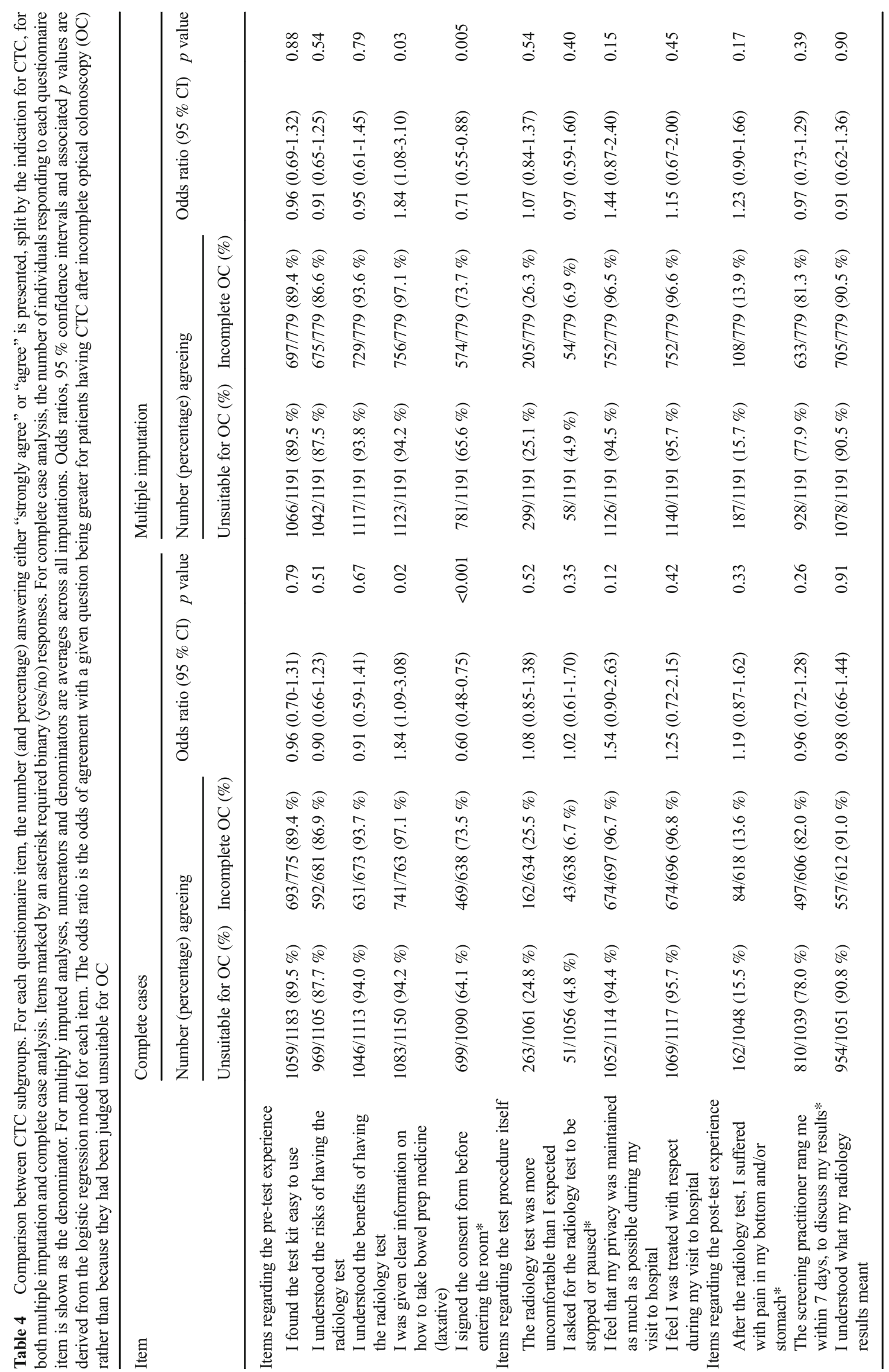


Fig. 2 Responses to the question "The test was more uncomfortable than I expected" for all respondents (top panel) and those who had undergone CTC after a previous attempt at colonoscopy (bottom panel). Overall, respondents were more likely to agree that CTC was more uncomfortable than they had expected, whereas the opposite was true for those who had undergone CTC following colonoscopy
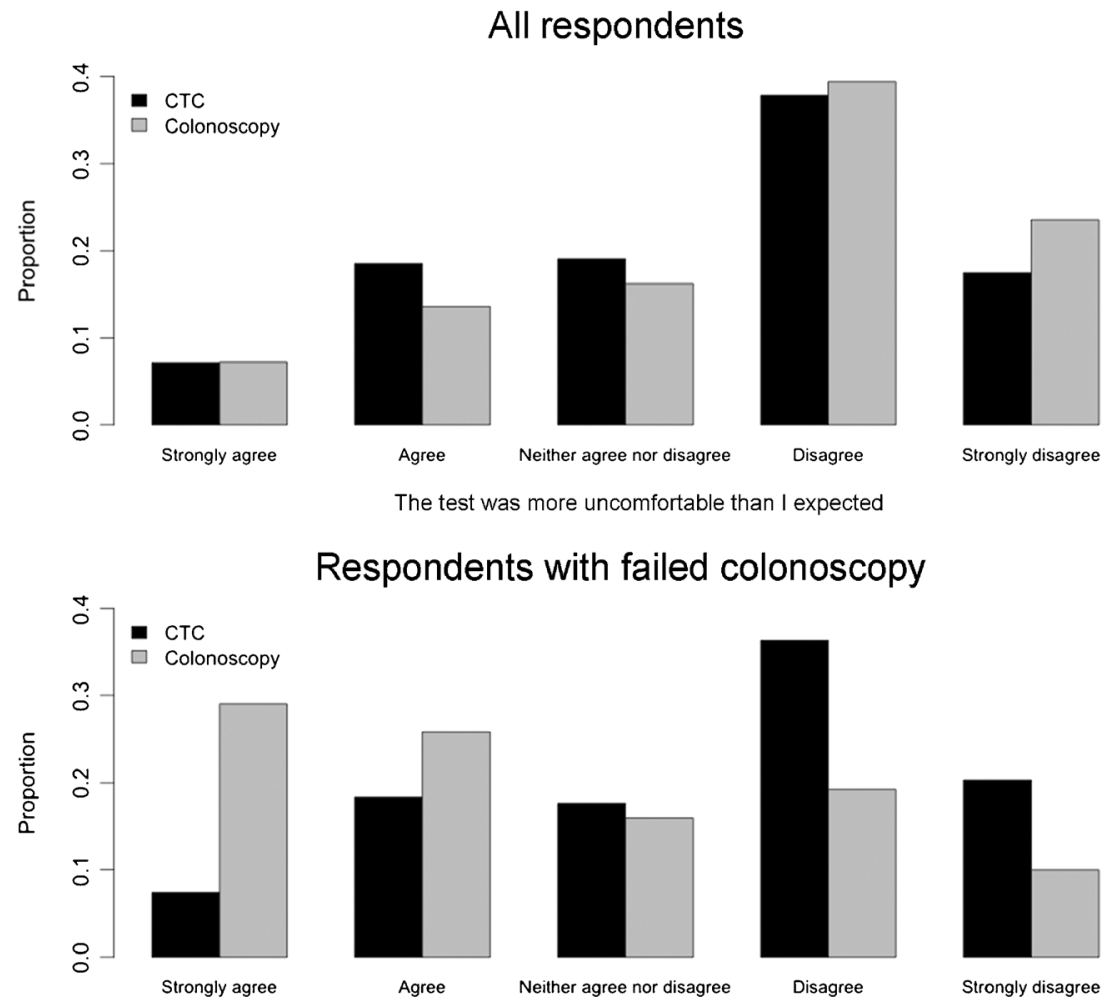

Respondents with failed colonoscopy
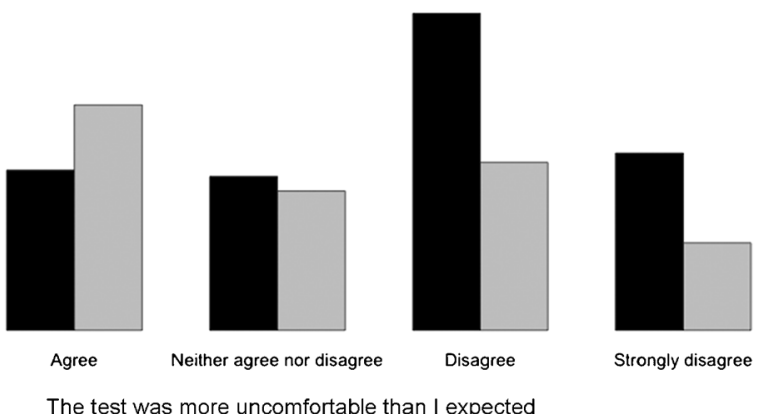

The test was more uncomfortable than I expected
These data show the "real-world" acceptability between the two tests, when employed as per current practice recommendations. It is extremely reassuring that although CTC is specifically reserved for individuals who are challenging to investigate (either because colonoscopy is contraindicated or it has already failed), overall acceptability and safety of the test is high. Indeed, differences from colonoscopy (which is performed for the majority of patients, most of whom will be healthy) were small and all $<5 \%$.

At first sight, our findings regarding patient comfort are somewhat surprising. Screenees were more likely to report that CTC was unexpectedly uncomfortable than colonoscopy, although the absolute difference was small $(\sim 5 \%)$. This is surprising, since CTC is generally viewed as the less invasive procedure. However, the questionnaire does not record opinions regarding absolute test-related discomfort-only relative discomfort in comparison to expectations. Respondents may have had an unrealistic impression of CTC, leading to any level of discomfort being greater than expected. Conversely, expectations of colonoscopy may have been for substantial discomfort. A previous randomised trial of CTC versus colonoscopy showed that participants expected significantly greater burden from colonoscopy than from CTC [18]. We, therefore, suspect that expectations of CTC were for minimal discomfort, and even fairly mild symptoms may have breached this threshold. Additionally, those undergoing colonoscopy are frequently offered sedoanalgesia, a factor which might further mitigate any differences in test discomfort, although we found that unexpected discomfort was more common at CTC even when compared to the subgroup of individuals who did not report having sedation for their colonoscopy (Table 3). This may again be due to expectations; screenees probably expect unsedated colonoscopy to be uncomfortable, and they are generally a self-selecting, motivated, and otherwise healthy cohort [28].

Nonetheless, a similar proportion of screenees requested that CTC be stopped or paused to colonoscopy (irrespective of use of sedation), which implies similar levels of absolute test-related discomfort. One contributing factor might be inherent differences between individuals undergoing CTC and colonoscopy. For example, those undergoing CTC were older than those having colonoscopy (albeit only by approximately 6 months), and more likely to be female. Furthermore, by definition CTC was only performed when colonoscopy was incomplete or deemed unsuitable initially. Such individuals may be particularly prone to finding any colonic procedure uncomfortable. It is important to emphasise that this is the precise manner in which CTC is generally employed in FOBt-based screening programmes; therefore, our data directly reflect clinical practice. We were unable to demonstrate an effect of reduced-laxative preparation on patient comfort, perhaps because the questionnaire only records comfort during or after the CTC procedure rather than symptoms from bowel purgation itself, or underpowering. 
Fig. 3 Funnel plots depicting variation between screening centres for the three "discomfortrelated" measures of CTC. Each circle represents the response for a given screening centre, plotted against the number of respondents at that centre, for (a) greater than expected discomfort, (b) asking for the test to be stopped or paused, and (c) rectal or abdominal pain after the test. Dashed and dotted lines represent $95 \%$ and $99.9 \%$ control limits, respectively a

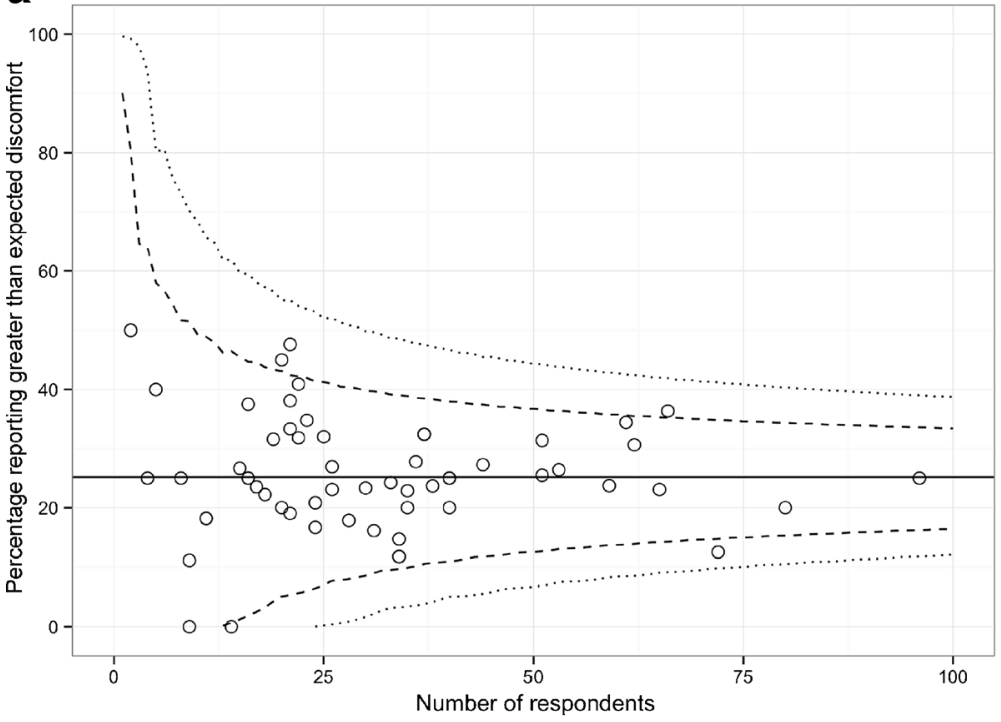

b

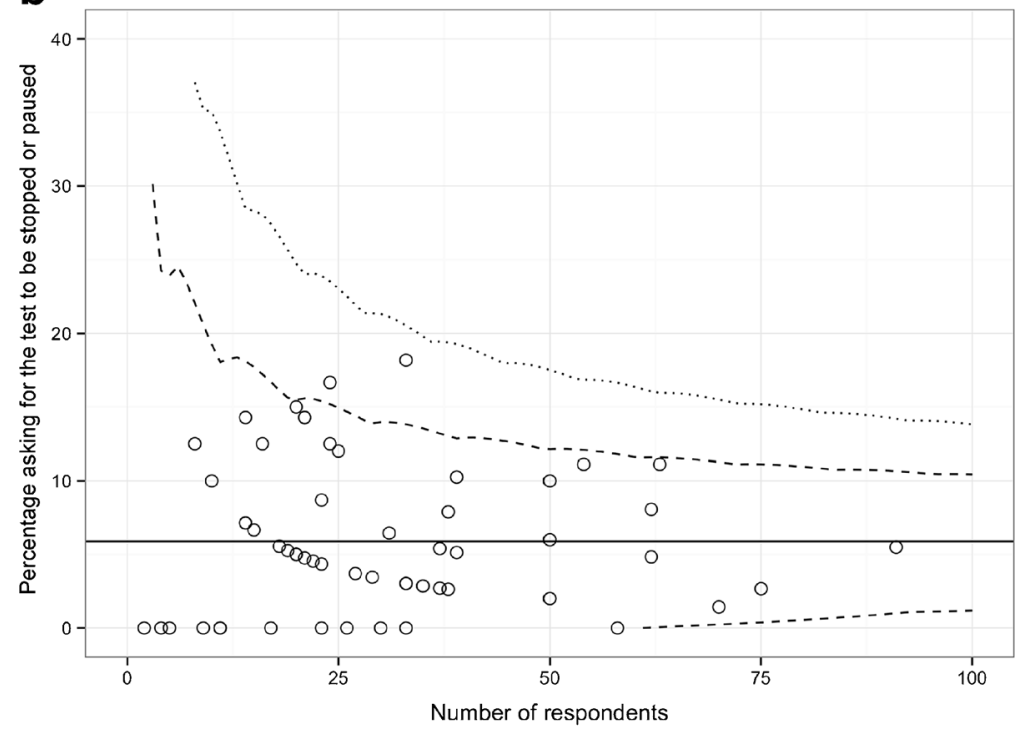

C

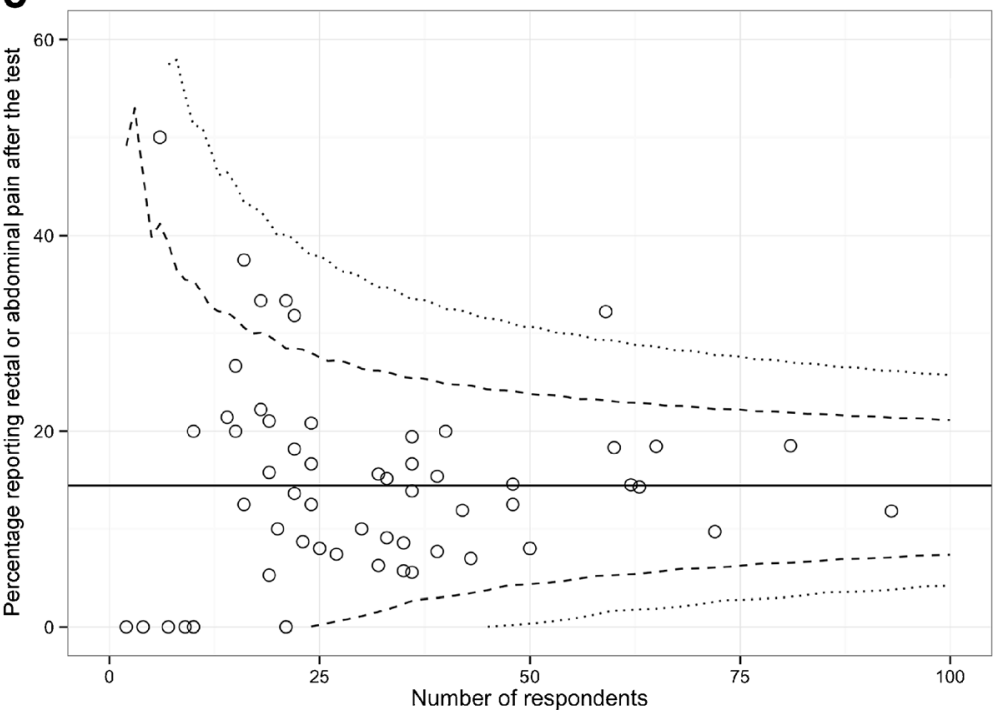


Importantly, individuals who had colonoscopy prior to CTC (i.e. failed endoscopy) had high levels of discomfort at colonoscopy, but tolerated CTC significantly better; indeed, as well as those in whom CTC was used as a first-line test after positive FOBt. We hope that this finding will allow clinicians to reassure screenees who have had a difficult experience at colonoscopy that they are likely to find CTC tolerable.

CTC generated relatively few direct complications, as expected for such a safe procedure. While the complication rate of CTC itself was lower than for colonoscopy, this difference disappeared once considering downstream complications due to further tests precipitated by CTC. However, complications arising after CTC were not serious: No patient had perforation as a result of CTC (either directly or downstream), whereas perforation occurred at a rate of approximately 1 in 1900 following colonoscopy, comparable with existing literature [26, 29, 30].

Strengths of this study include the large sample size, high response rate $(79 \%)$, and multicenter nature with national coverage. Although missing data were noted, this was not problematic. However, our study has limitations. As noted above, the participants were not randomised, and therefore, differences may be due to variation between the tested populations rather than directly reflecting test characteristics. Arguably, this is unimportant from a clinical perspective: CTC was employed precisely as is recommended by international consensus $[10,11]$, meaning our results reflect clinical practice. However, we would strongly caution against using these data to argue that CTC or colonoscopy is the bettertolerated test when employed as a primary screening tool for asymptomatic subjects: CTC was reserved for those who are unsuitable for colonoscopy (roughly 60:40 split of contraindications to colonoscopy vs. incomplete colonoscopy) and was well-tolerated overall, supporting its use in this role. It is impossible to know whether or not these potentially vulnerable individuals would have found colonoscopy more or less tolerable than CTC. Our data are likely generalizable to mass screening programmes based on FOBt/FIT, but will not reflect the relative patient experience of colonoscopy or CTC when employed as first-line screening tests.

Although test complications are recorded centrally, we cannot be certain that all relevant complications were captured and uploaded successfully. Additionally, due to the large size of the dataset, most comparisons were highly statistically significant, even when absolute differences were small. It is, therefore, largely a matter of judgement whether or not the differences we report here are clinically meaningful or not.

In summary, both CTC and colonoscopy have high levels of satisfaction when used to investigate a positive FOBt result in a national CRC screening programme. Although screenees directed to CTC are a different population to those having colonoscopy, overall test tolerability was good, with only a small increase in test discomfort and no difference in post- procedural pain or complication rates. Screenees requiring CTC after incomplete colonoscopy tolerated CTC extremely well. Individuals requiring CTC may require more detailed information regarding expected procedural discomfort.

Acknowledgments The scientific guarantor of this publication is Andrew Plumb. The authors of this manuscript declare relationships with the following companies: CJR: Has received unrestricted educational grants from Aquilant Endoscopy, Cook Medical Products and Olympus Endoscopy. SAT: Has been remunerated for research and development advice by Medicsight, a software company developing computer-assisted detection for CTC. SH: Has been remunerated for research and development advice by Medicsight, a software company developing computerassisted detection for CTC, and provides non-remunerated research and development advice for iCAD inc. The current study was supported by a programme grant from Cancer Research UK (C1418/A14134). The study was conducted in part at University College London / University College London Hospitals, which receive a proportion of funding from the National Institute for Health Research Biomedical Research Centre funding scheme. Neither Cancer Research UK nor the NIHR were involved in the design of this study; the collection, analysis, or interpretation of the results; in the writing of the manuscript; or in the decision to submit for publication. The views expressed in this article are those of the authors, and not necessarily those of Cancer Research UK, the NIHR or the UK Department of Health. One of the authors has significant statistical expertise. Institutional Review Board approval was obtained. Written informed consent was waived by the Institutional Review Board. Methodology: retrospective, observational study, multicenter study. Some of the colonoscopy data have been submitted for publication separately to Endoscopy.

Open Access This article is distributed under the terms of the Creative Commons Attribution 4.0 International License (http:// creativecommons.org/licenses/by/4.0/), which permits unrestricted use, distribution, and reproduction in any medium, provided you give appropriate credit to the original author(s) and the source, provide a link to the Creative Commons license, and indicate if changes were made.

\section{References}

1. Robert G, Cornwell J, Brearley S, et al (2011) What matters to patients? Developing the evidence base for measuring and improving patient experience. Department of Health and NHS Institute for Innovation and Improvement

2. Francis R (2013) Report of the Mid Staffordshire NHS Foundation Trust Public Inquiry

3. Benson VS, Atkin WS, Green J et al (2012) Toward standardizing and reporting colorectal cancer screening indicators on an international level: The International Colorectal Cancer Screening Network. Int J Cancer 130:2961-2973

4. Benson VS, Patnick J, Davies AK et al (2008) Colorectal cancer screening: a comparison of 35 initiatives in 17 countries. Int $\mathrm{J}$ Cancer 122:1357-1367

5. Schreuders EH, Ruco A, Rabeneck L, et al (2015) Colorectal cancer screening: a global overview of existing programmes. Gut

6. Hewitson P, Glasziou P, Irwig L, Towler B, Watson E (2007) Screening for colorectal cancer using the faecal occult blood test, Hemoccult. Cochrane Database Syst Rev (1)

7. Pickhardt PJ, Choi JR, Hwang I et al (2003) Computed tomographic virtual colonoscopy to screen for colorectal neoplasia in asymptomatic adults. N Engl J Med 349:2191-2200 
8. Johnson CD, Chen M-H, Toledano AY et al (2008) Accuracy of CT colonography for detection of large adenomas and cancers. N Engl J Med 359:1207-1217

9. Plumb AA, Halligan S, Pendse DA, Taylor SA, Mallett S (2014) Sensitivity and specificity of CT colonography for the detection of colonic neoplasia after positive faecal occult blood testing: systematic review and meta-analysis. Eur Radiol

10. Spada C, Stoker J, Alarcon O et al (2014) Clinical indications for computed tomographic colonography: European Society of Gastrointestinal Endoscopy (ESGE) and European Society of Gastrointestinal and Abdominal Radiology (ESGAR) Guideline. Endoscopy 46:897-915

11. Spada C, Stoker J, Alarcon O et al (2015) Clinical indications for computed tomographic colonography: European Society of Gastrointestinal Endoscopy (ESGE) and European Society of Gastrointestinal and Abdominal Radiology (ESGAR) Guideline. Eur Radiol 25:331-345

12. Taylor SA, Burling DN, Patnick J (2012) Guidelines for the use of imaging in the NHS Bowel Cancer Screening Programme: Second Edition: NHS Cancer Screening Programmes. Available at: www. cancerscreening.nhs.uk/bowel/publications/nhsbcsp05.pdf. Accessed 28 Jan 2015

13. Lin OS, Kozarek RA, Gluck M et al (2012) Preference for colonoscopy versus computerized tomographic colonography: a systematic review and meta-analysis of observational studies. J Gen Intern Med 27:1349-1360

14. Taylor SA, Halligan S, Saunders BP, Bassett P, Vance M, Bartram CI (2003) Acceptance by patients of multidetector CT colonography compared with barium enema examinations, flexible sigmoidoscopy, and colonoscopy. AJR Am J Roentgenol 181:913921

15. Gluecker TM, Johnson CD, Harmsen WS et al (2003) Colorectal cancer screening with CT colonography, colonoscopy, and doublecontrast barium enema examination: prospective assessment of patient perceptions and preferences. Radiology 227:378-384

16. Stoop EM, de Haan MC, de Wijkerslooth TR, et al (2011) Participation and yield of colonoscopy versus non-cathartic CT colonography in population-based screening for colorectal cancer: a randomised controlled trial. Lancet Oncol

17. Atkin W, Dadswell E, Wooldrage K et al (2013) Computed tomographic colonography versus colonoscopy for investigation of patients with symptoms suggestive of colorectal cancer (SIGGAR): a multicentre randomised trial. Lancet 381:1194-1202
18. de Wijkerslooth TR, de Haan MC, Stoop EM, et al (2011) Burden of colonoscopy compared to non-cathartic CT-colonography in a colorectal cancer screening programme: randomised controlled trial. Gut

19. von Wagner C, Ghanouni A, Halligan S et al (2012) Patient acceptability and psychologic consequences of CT colonography compared with those of colonoscopy: results from a multicenter randomized controlled trial of symptomatic patients. Radiology 263: 723-731

20. Logan RFA, Patnick J, Nickerson C, Coleman L, Rutter MD, von Wagner C (2012) Outcomes of the Bowel Cancer Screening Programme (BCSP) in England after the first 1 million tests. Gut 61(10):1439-1446

21. Plumb AA, Halligan S, Taylor SA, Burling D, Nickerson C, Patnick J (2013) CT colonography in the English Bowel Cancer Screening Programme: national survey of current practice. Clin Radiol 68(5): 479-487

22. Joint Advisory Group on GI Endoscopy (2013) BCSP Guidelines Accreditation of screening colonoscopists. Royal College of Physicians. Available from: http://www.saas.nhs.uk/Downloads. aspx. Accessed 1 Mar 2014

23. van Buuren S, Groothuis-Oudshoorn K (2011) mice: Multivariate imputation by chained equations in R. J Stat Softw 45:1-67

24. Rubin DB (1987) Multiple imputation for nonresponse in surveys. J. Wiley \& Sons, New York

25. Spiegelhalter DJ (2005) Funnel plots for comparing institutional performance. Stat Med 24:1185-1202

26. Lee TJW, Rutter MD, Blanks RG et al (2012) Colonoscopy quality measures: experience from the NHS Bowel Cancer Screening Programme. Gut 61:1050-1057

27. Plumb AA, Halligan S, Nickerson C et al (2014) Use of CT colonography in the English Bowel Cancer Screening Programme. Gut 63:964-973

28. Ball AJ, Rees CJ, Corfe BM, Riley SA (2015) Sedation practice and comfort during colonoscopy: lessons learnt from a national screening programme. Eur J Gastroenterol Hepatol 27:741-746

29. Stock C, Ihle P, Sieg A, Schubert I, Hoffmeister M, Brenner H (2013) Adverse events requiring hospitalization within 30 days after outpatient screening and nonscreening colonoscopies. Gastrointest Endosc 77:419-429

30. Pox CP, Altenhofen L, Brenner H, Theilmeier A, Von Stillfried D, Schmiegel W (2012) Efficacy of a nationwide screening colonoscopy program for colorectal cancer. Gastroenterology 142:14601467, e2 